\title{
GROUPS DEFINED ON IMAGES IN FLUID DIFFUSION
}

\author{
A. J. BRACKEN ${ }^{1}$, H. S. GREEN ${ }^{1,2}$ AND L. BASS $^{1}$
}

(Received 23 April 1987; revised 1 June 1987)

\begin{abstract}
A method based on the method of images is described for the solution of the linear equation modelling diffusion and elimination of substrate in a fluid flowing through a chemical reactor of finite length, when the influx of substrate is prescribed at the point of entry and Danckwerts' zero-gradient condition is imposed at the point of exit. The problem is shown to be transformable to an equivalent problem in heat conduction. Associated with the images appearing in the method of solution is a sequence of functions which form a vector space carrying a representation of the Lie group $S O(2,1)$ generated by three third-order differential operators. The functions are eigenfunctions of one of these operators, with integer-spaced eigenvalues, and they satisfy a third-order recurrence relation which simplifies their successive determination, and hence the determination of the Green's function for the problem, to any desired degree of approximation. Consequently, the method has considerable computational advantages over commonly used methods based on the use of Laplace and related transforms. Associated with these functions is a sequence of polynomials satisfying the same third-order differential equation and recurrence relation. The polynomials are shown to bear a simple relationship to Laguerre polynomials and to satisfy the ordinary diffusion equation, for which $S O(2,1)$ is therefore revealed as an invariance group. These diffusion polynomials are distinct from the well-known heat polynomials, but a relationship between them is derived. A generalised set of diffusion polynomials, based on the associated Laguerre polynomials, is also described, having similar properties.
\end{abstract}

\section{Introduction}

The method of images was developed originally by Maxwell and Thompson to obtain solutions of Laplace's equation for problems in electrostatics with boundary conditions prescribed on any set of plane or spherical surfaces. It had a simple extension, not only to static problems in heat conduction, diffusion and

\footnotetext{
${ }^{1}$ Department of Mathematics, University of Queensland, St Lucia, Queensland, 4067.

${ }^{2}$ Department of Mathematical Physics, University of Adelaide, South Australia, 5001.

(C) Copyright Australian Mathematical Society 1988, Serial-fee code 0334-2700/88
} 
viscosity where the solution of Laplace's equation with equivalent boundary conditions is also required, but also to the corresponding time-dependent problems, where solutions of the heat equation are required. The power of this method was very well illustrated by Sommerfeld [19] in his well known treatise.

The method of images also has a variety of applications [13] to problems involving diffusion in moving fluids, and the generalisation is very easy when there is no flux through the boundaries on which the boundary conditions are specified. However, where such flux does exist, the method is not applicable in its usual form. In consequence, in most of the rather extensive literature on fluid reactors (see e.g., Wen and Fan [23]; Parulekar and Ramakrishna [16]) and diffusion in biological systems (Perl and Chinard [17]; Roberts and Rowland [18]), there is a reliance on methods involving Laplace and related transforms, where the solutions obtained do not provide much insight into the process of diffusion in that context and, since they involve functions of at least two variables, are not very convenient from the computational point of view.

The use of the method of images makes it possible to exhibit the solution of a partial differential equation, satisfying appropriate boundary conditions, as a sum of contributions from sources localised either on the boundary of the region of interest or outside the region. Usually the sources on the boundary are physical sources, and the contribution of these sources to the solution is just what would be expected in the absence of the boundary. The contributions of the image sources outside the boundary satisfy the differential equation independently, and the positions and strengths of these sources are chosen so that the complete solution satisfies the boundary conditions. The method has the obvious advantage that the contribution of each source to the solution is quite simple and easily visualised, and the way in which the entire solution is built up from these simple components is made apparent. In the applications to diffusion, it is also usually evident that the contributions from distant sources will be negligible within the time span of interest, or even within the time needed to approach a steady state. In fact, as we shall demonstrate below, it often happens that, for computational purposes, the sum of contributions from image sources outside the boundary may be approximated very closely by one or two terms.

In this paper, we shall present a modification of the method of images which is directly applicable to problems of diffusion in moving fluids where there is a flux through the boundary, and is, we believe, of interest for a number of reasons. From the practical point of view, it retains the advantages which we have just described as characteristic of the method of images, and throws new light on the effect of the boundary conditions on the solutions obtained. But, in addition, the explicit solutions obtained are of considerable mathematical interest because of their relationship to a Lie algebra of third-order difference operators. We 
are thus extending the repertoire of uses of special functions in relation to the differential equations of mathematical physics.

There is a close analogy between diffusion and heat conduction, and it might be expected that there should be some relation between this work and various studies of the heat equation based on group-theoretical considerations (see Bluman and Cole, [5]) dating from that of Sophus Lie himself. Although, as we show in an Appendix (Section 6), the problem that we consider here can in fact be transformed to an equivalent problem in heat conduction (or ordinary diffusion), we could find no such relation. This is mainly because in these earlier works, only differential operators of first or second order seem to have been considered, so that the possibility of representing group generators in terms of third-order operators did not arise there. As appropriate, we shall point out connnections between this apparently new representation and well known others, but we shall also draw attention to some interesting special features of the diffusion functions and polynomials, both of which form representation spaces of the group in question.

We begin with a description of one of the more important applications, which is to a well known and somewhat controversial problem in the modelling of chemical reactors, or their biological equivalents. A fluid containing a substrate in solution passes through a semi-permeable wall or membrane into a reactor, where the substrate is removed by chemical reaction with another substance which is either insoluble or otherwise unable to penetrate the wall. The fluid finally passes through another semi-permeable boundary out of the reactor. The problem is to determine the concentration $c$ of the substrate as a function of position and time, and in particular the outflux of substrate from the reactor, given the influx into it, that is, input-output relations.

The geometry of the reactor is usually cylindrical, and we shall assume a uniform cross-section, so that the velocity $v$ of the fluid is independent of the longitudinal coordinate $x$. If $D$ is the eddy coefficient of diffusion within the reactor, the flux $j(x, t)$ of substrate with concentration $c(x, t)$ is

$$
j=v c-D \partial c / \partial x .
$$

This equation is unaffected by averaging over the cross-section, and the equation of continuity may then also be written in the one-dimensional form

$$
\partial c / \partial t+\partial j / \partial x=-\rho c
$$

where $\rho$ is the reaction rate constant. (We consider only first-order reaction kinetics.) Thus the concentration satisfies

$$
\partial c / \partial t+(\partial / \partial x)[v c-D \partial c / \partial x]=-\rho c .
$$

A similar equation holds for the solution outside the reactor, where, however, $\rho=0$, and the eddy coefficient of diffusion is often very much smaller, owing 
to the absence of the reacting material. But, provided that $D$ is everywhere finite, there should be little disagreement about the boundary conditions to be applied: both $c$ and $j$ must be continuous through the reactor boundary. There was a problem to be settled when $D$ was assumed to vanish outside the reactor, reminiscent of the problem of appropriate boundary conditions for an inviscid fluid, since there also the order of the differential equation is reduced in the limit considered. The generally accepted boundary conditions in this instance are those given by Danckwerts [7], and confirmed by the more detailed analyses of Wehner and Wilhelm [22] and van Cauwenberghe [6]. The flux $j$ is necessarily continuous at all boundaries; also the concentration is continuous across the exit face of the reactor and its derivative $\partial c / \partial x$ then has to vanish at the internal surface of that face. However, when $D$ vanishes outside the reactor, there has to be a discontinuity in the concentration at the point of entry. Since other authors [22], [6] have adequately considered this limit, we shall not comment on this rather surprising asymmetry, but draw attention to the more surprising experimental results of Deckwer and Mählmann [8], [9], tending to show that localised Danckwerts' boundary conditions are satisfied even at an internal boundary between two reactor segments, in each of which the eddy coefficient of diffusion is quite large. In particular, there is a discontinuity of concentration across such a boundary and the concentration has a vanishing derivative at the point of exit from each reactor segment. We hope to show how the method of images illuminates these and similar experimental findings, which have led to much debate in the literature (Wicke [24], Sundaresan, Amundson and Aris [20]).

\section{Relation between source and image for a fluid in motion}

We consider a fluid reactor characterised by the constants $v, D$ and $\rho$ appearing in (1.3), and with entry and exit boundaries at $x=0$ and $x=L$ respectively. We shall seek a solution of (1.3) satisfying Danckwerts' condition $\partial c / \partial x=0$ at $x=L$, which we accept as appropriate when $v>0$, and having a prescribed flux $j(0, t)$ at $x=0$. This will be done by formulating such a solution as an integral which takes account of the boundary conditions. The most general solution can be regarded as a sum of this solution and a complementary 'anti-Danckwerts' solution satisfying $\partial c / \partial x=0$ at $x=0$, and with a specified flux $j(L, t)$ at $x=L$.

The desired solution can be written in the form

$$
c(x, t)=\int_{-\infty}^{t} G\left(x, t-t^{\prime}\right) j\left(0, t^{\prime}\right) d t^{\prime},
$$

where $G(x, t)$ is a Green's function, satisfying (1.3) for $t>0$ and $0<x<L$. It is convenient to define $G(x, t)=0$ for $t<0$, and the upper limit of integration 
can then be replaced by infinity, if so desired. Furthermore, we usually suppose that $j(0, t)=0$ for $t<0$ (and that the reactor is initially free of substrate: $c(x, 0)=0$ ) so that the lower limit can be replaced by 0 . If this solution $(2.1)$ is to satisfy, for arbitrary $j(0, t)$, the condition $\partial c / \partial x=0$ at $x=L$ for $t>0$, then

$$
G^{\prime}(L, t)=0 \text { for } t>0,
$$

where $G^{\prime}=\partial G / \partial x$. Furthermore, (2.1) implies that $G(x, t)$ describes the concentration resulting from a singular source of flux localised at $t=0$ on the boundary, $j(0, t)=\delta(t)$, so that if

$$
j_{G}(x, t)=v G(x, t)-D G^{\prime}(x, t)
$$

is the flux associated with $G$, we must have

$$
\lim _{x \rightarrow 0+} j_{G}(x, t)=\delta(t)
$$

We shall refer to such a singular source of flux at $x=0$ as a primary source for the Green's function, and construct a solution in the form of a series

$$
G(x, t)=\sum_{k=0}^{\infty} G_{k}(x, t),
$$

where $G_{0}(x, t)$ is a solution for the primary source in a semi-infinite reactor (occupying $x \geq 0$ ), satisfying (2.4) but not (2.2). The remaining terms in (2.5) are solutions for a set of image sources located at the points

$$
y_{k}=\frac{1}{2} L\left[1-(-1)^{k}(2 k+1)\right]
$$

$(k=1,2, \ldots)$, so that, for odd $k, y_{k+1}$ is the image of $y_{k}$ by reflection in the plane through $x=0$, and for even $k$ it is the image of $y_{k}$ by reflection in the plane through $x=L$. The sources, though localised, are not point sources in the usual sense, and the relation between a source and its image is by no means as simple as in other applications of the method of images.

To determine that relation between source and image, we write

$$
\begin{aligned}
G_{k}(x, t) & =a(x, t) f_{k}\left(x-y_{k}+v t, t\right), \quad k \text { even, } \\
G_{k}(x, t) & =a(x, t) f_{k}\left(y_{k}-x+v t, t\right), \quad k \text { odd, } \\
a(x, t) & =\exp \left[-\rho t+\frac{1}{2} v(x-v t) / D\right] / \sqrt{(4 \pi D t)},
\end{aligned}
$$

noting that, for any $k, G_{k}(x, t)$ is a solution of (1.3) provided that $f_{k}(x, t)$ is of the form

$$
f_{k}(x, t)=-(2 D / v)(\partial / \partial x)\left[\exp \left(\frac{1}{2} v x / D\right) g_{k}(x, t)\right],
$$

where $g_{k}(x, t) / \sqrt{t}$ satisfies the diffusion equation without convection or elimination terms, that is, (1.3) with $v=\rho=0$. The expression (2.8) has been chosen because, as we shall proceed to show, the $g_{k}(x, t)$ thus defined satisfy a variety of relations much simpler than the $f_{k}(x, t)$. For simplicity, we shall write $f_{k}$ or 
$f_{k}(x)$ for $f_{k}(x, t)$ and $g_{k}$ or $g_{k}(x)$ for $g_{k}(x, t)$ in the following. Note that these functions must be defined for all $x \geq 0$.

It can be seen from (2.5) that the boundary conditions (2.2) and (2.4) will both be satisfied by $G(x, t)$ provided that, for $t>0$,

$$
\begin{gathered}
G_{k}^{\prime}(L, t)+G_{k+1}^{\prime}(L, t)=0, \quad k \text { even } \\
j_{k}(0, t)+j_{k+1}(0, t)=0, \quad k \text { odd }
\end{gathered}
$$

and provided also that

$$
\lim _{x \rightarrow 0+} j_{0}(x, t)=\delta(t)
$$

Here the primes again denote differentiation with respect to $x$, and

$$
j_{k}(x, t)=v G_{k}(x, t)-D G_{k}^{\prime}(x, t), \quad k=0,1,2, \cdots
$$

is the flux associated with $G_{k}$. Conditions (2.9) require that

$$
\frac{1}{2}(v / D)\left(f_{k}+f_{k+1}\right)+f_{k}^{\prime}-f_{k+1}^{\prime}=0
$$

for all $k$, for all $t>0$ and for all $x \geq 0$. After the substitution of (2.8), and assuming that $f_{k}(x)$ and $g_{k}(x)$ vanish sufficiently quickly as $x \rightarrow \infty$, this equation can be integrated twice with the results

$$
g_{k+1}^{\prime}(x)=g_{k}^{\prime}(x)+(v / D) g_{k}(x)
$$

and then

$$
g_{k+1}(x)=g_{k}(x)-(v / D) \int_{x}^{\infty} g_{k}(y) d y
$$

This leaves $g_{0}$ or equivalently $G_{0}$ to be determined. As shown in the Appendix (Section 6), $G_{0}$ is given by (6.17) and hence, from (2.7) and (2.8),

$$
g_{0}(x)=E(x)=(v / D) \int_{x}^{\infty} \exp \left(-y^{2} / 4 D t\right) d y,
$$

also expressible in terms of the error function $\operatorname{erfc}(z)$. Together, (2.14) and (2.15) define a series of functions of considerable mathematical interest, as we shall demonstrate in the next section. For the present, we note that

$$
\begin{gathered}
g_{1}(x)=(1+v x / D) E(x)-\left(2 v^{2} t / D\right) e(x), \\
e(x)=\exp \left(-x^{2} / 4 D t\right),
\end{gathered}
$$

and, in general,

$$
g_{k}(x)=p_{k}(x) E(x)-2 v t q_{k}(x) e(x)
$$

where $p_{k}(x)$ and $q_{k}(x)$ are polynomials of degree $k$ and $k-1$ respectively, with parametric dependence on $t$.

It also follows from (6.17) that

$$
j_{0}(x, t)=(x / t) \exp \left[-\rho t-(x-v t)^{2} / 4 D t\right] / \sqrt{(4 \pi D t)},
$$


which satisfies, for any sufficiently smooth $F(t)$ and any positive $\varepsilon$, no matter how small,

$$
\lim _{x \rightarrow 0+} \int_{0}^{\varepsilon} j_{0}(x, t) F(t) d t=F(0)
$$

This can be seen quite easily after expanding $F$ in powers of $t$ and, for the leading term, changing the variable of integration from $t$ to $u=x / \sqrt{(4 D t)}$. Thus (2.10) holds as required.

Owing to the increasing distance of successive images from the interval $0 \leq$ $x \leq L$ where the solution is to be determined, and the very rapid rate of decrease of both $e(x)$ and $E(x)$ with increasing positive $x$, it is now evident that, as stated in the Introduction, only the terms $G_{0}(x, t)$ and $G_{1}(x, t)$ of the series (2.5) will normally contribute significantly to the value of $G(x, t)$. The main exception is for very short reactors, where it may be necessary to calculate quite a number of terms of the series; however, our subsequent analysis will provide a recurrence relation for the $g_{k}(x)$, and incidentally for the $p_{k}(x)$ and $q_{k}(x)$ as well, so that this is not a computational difficulty. Another apparent exception to the possibility of replacing the series (2.5) with its first few terms is for large values of $t$; however, in this instance $G(x, t)$ as a whole becomes quite small and its contribution to $c(x, t)$ in $(2.1)$ is likely to be negligible.

An example of the application of the formula (2.1) is shown in Figure 1, which illustrates the progress of a delta-function source of flux originating at $x=0$ on the left of the Figure, at time $t=0$, through a series of reactor segments of the type studied by Deckwer and Mählmann [8], [9] in the experiments we have already referred to, except that for simplicity, we have ignored the effects of elimination and set $\rho=0$ everywhere. Our illustration includes some narrow boundary layers of semi-permeable material which, we suppose, enclose or separate reactor material at different densities.

In these boundary layers, the effective coefficient of diffusion is certainly very much smaller than in the reactor segments, where turbulent mixing is responsible for Peclet numbers (defined in any region with length $\lambda$ and coefficient of diffusion $D$ as $\lambda v / D$, where $v$ is the convective velocity, the same for all regions) equal to 13.1, 7.4 and 0.55 in the left, central and right segments respectively. The Peclet numbers in the boundary layers were not measured in the experiments quoted, but the predicted concentration curves are not sensitive to the precise values assigned there, provided that the thickness of the layers is small. Indeed, the curves obtained for any reasonable values are almost the same as those obtained when infinite Peclet numbers (vanishing diffusion coefficients) are adopted for the boundary layers. A typical curve of the latter type is shown in Figure 1. The presence of such boundary layers means therefore that Danckwerts' boundary conditions are an excellent approximation for each reactor segment, and there will in practice be an apparent discontinuity in the concentration across each 


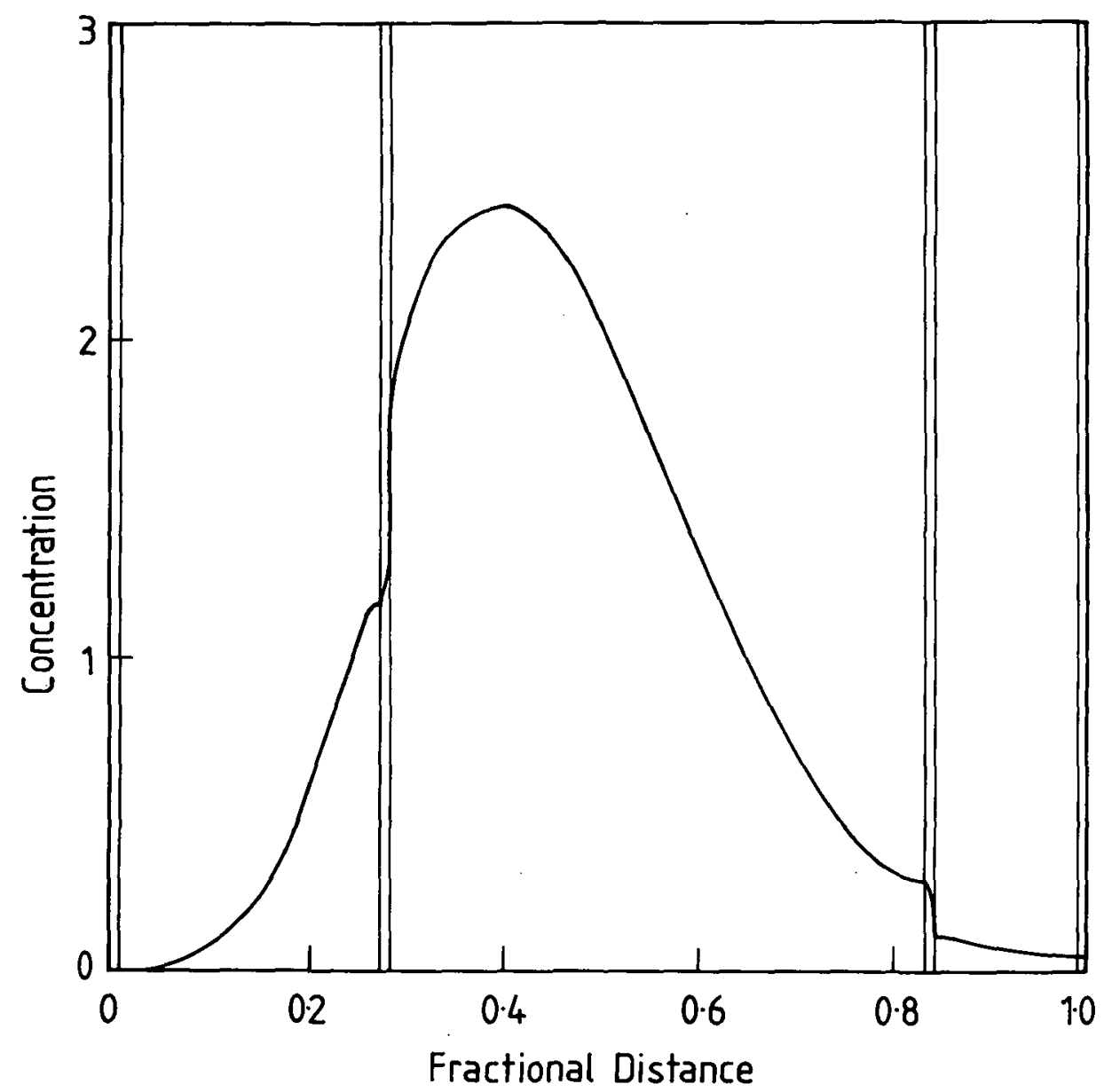

FIGURE 1. Substrate distribution at time $t=0.4 L / v$ corresponding to a delta-function primary source of flux at time $t=0$, showing apparent discontinuities at internal reactor boundaries. The Peclet numbers in the three reactor segments from left to right are 13.1, 7.4 and $\mathbf{0 . 5 5}$, while all four boundary layers have Peclet number $\infty$. In all regions $\rho=0$.

boundary layer, and an almost vanishing derivative at the exit from each reactor segment. In making these calculations, the delta-function source was used only for the left segment of the reactor; the source for each subsequent segment was obtained by computing and storing the flux, as a function of time, at the end of the preceding segment. The curve corresponding to Figure 1 for a steady state 
$(\partial c / \partial t=0)$ is even more easily calculated, shows similar characteristics, and approximates very closely to a curve published by Deckwer and Mählmann [8, Figure 5] and shown to be consistent with experiment. We intend to publish a more detailed account of boundary layers elsewhere [4]; our object here in this matter is only to provide a simple and comprehensible justification for the common practice of using Danckwert's boundary conditions in circumstances where the eddy coefficient of diffusion is not negligible inside or outside the reactor.

\section{Properties of the diffusion functions}

In this section we shall outline the essential properties of the functions $g_{k}(x)$ defined by (2.14) and (2.15), and the associated polynomials $p_{k}(x)$ and $q_{k}(x)$ appearing in (2.17), which we shall call diffusion polynomials. They are distinct from the well-known heat polynomials (see Widder [25]), although, as we shall show, the $p_{k}(x)$ satisfy an equation similar to the heat equation. We shall also show incidentally that $g_{k}(x), p_{k}(x)$ and $q_{k}(x)$ all satisfy a third order recurrence relation which greatly assists their computation. To simplify the notation, we choose units of length and time such that

$$
v=D=\frac{1}{2} \text {. }
$$

Arbitrary units may be restored in subsequent formulae by replacing $x$ and $t$ with the dimensionless variables $v x / D$ and $2 v^{2} t / D$.

We first note that the differential recurrence relation (2.13) is satisfied by the Laguerre polynomials $L_{k}(-x)$, which can be defined by means of $L_{0}(x)=1$ and the recurrence relation

$$
x L_{k}(x)=(2 k+1) L_{k}(x)-(k+1) L_{k+1}(x)-k L_{k-1}(x)
$$

(see Abramowitz and Stegun [1]), or by Rodrigues formula

$$
L_{k}(x)=e^{x}(d / d x)^{k}\left(x^{k} e^{-x}\right) / k !,
$$

from which (2.13) is easily derived. But they are not at all unique in this respect, and definite integrals involving $L_{k}(y-x)$ will also satisfy $(2.13)$. Since $L_{k}(0)=1$, it is easily verified in particular that the integral formula

$$
g_{k}(x)=\int_{x}^{\infty} \exp \left(-\frac{1}{2} y^{2} / t\right) L_{k}(y-x) d y
$$

will also satisfy (2.13) in the units adopted. Moreover, as the right side of (3.4) vanishes when $x \rightarrow \infty$, it will satisfy (2.14). Finally, since (3.4) reduces to (2.15) 
when $k=0$, the $g_{k}(x)$ defined by (3.4) are the same as those defined by (2.14) and (2.15).

By differentiation and integration by parts we infer from (3.4) that

$$
t g_{k}^{\prime}(x)+x g_{k}(x)=-\int_{x}^{\infty} \exp \left(-\frac{1}{2} y^{2} / t\right)(y-x) L_{k}(y-x) d y .
$$

Then, by using the recurrence relation (3.2) of the Laguerre polynomials, we have

$$
t g_{k}^{\prime}(x)+x g_{k}(x)=(k+1) g_{k+1}(x)-(2 k+1) g_{k}(x)+k g_{k-1}(x) .
$$

With the help of (2.13), the derivatives may be eliminated between this equation and a similar equation with $k$ replaced by $k+1$, yielding the third order difference equation

$$
(k+2) g_{k+2}=(x+3 k+4) g_{k+1}-(x-t+3 k+2) g_{k}+k g_{k-1} .
$$

With $g_{0}$ and $g_{1}$ given by (2.15) and (2.16), this relation gives an easy method of computation of as many of the $g_{k}(x)$ as required. It can also be used to determine the diffusion polynomials $p_{k}(x)$ and $q_{k}(x)$ appearing in (2.17), since both series must obviously satisfy the same recurrence relation. In this instance, the initial terms are given by

$$
\begin{array}{ll}
p_{0}(x)=1, & p_{1}(x)=x+1, \\
q_{0}(x)=0, & q_{1}(x)=1 .
\end{array}
$$

Since the recurrence relation (3.7) is of the third order, it has a third linearly independent solution, which is, however, related to the others since the equation is invariant under the successive transformations $k \rightarrow-(k+2), g_{k} \rightarrow g_{1-k}$ (so that, e.g., $\left.g_{-k} \rightarrow g_{k-1}\right), t \rightarrow-t$ and $x \rightarrow t-x$.

We next show that the functions $g_{k}(x)$ defined in (3.4) not only satisfy a differential equation of the third order, but that contiguous functions like $g_{k}$ and $g_{k+1}$ can be expressed in terms of one another by differential equations of the third order. For this purpose we differentiate (3.6) with respect to $x$ and simplify the result with the help of (2.13), obtaining

$$
t g_{k}^{\prime \prime}+x g_{k}^{\prime}=k\left(g_{k}-g_{k-1}\right)
$$

as an intermediate result; then the repetition of this process gives the differential equation of the third order in question:

$$
A g_{k} \equiv[(d / d x)+1][t(d / d x)+x] d g_{k} / d x=k g_{k},
$$

which also defines the linear differential operator $A$. As a differential equation, this belongs to a class solvable by Laplace transforms and which was, in fact, treated by Laplace (see Vandenberg and Deakin, [21]). The solution obtained in this way can be used to recover (3.4). From another point of view, (3.10) may be regarded as a generalisation of the confluent hypergeometric equation, though 
it, and its solution, are not among the generalisations which are in common use ([12], Ch. 4). But the equation has various properties in common with equations of hypergeometric type, including those which we have already noted: polynomial solutions, and the existence of recurrence relations (albeit of the third order) and differential recurrence relations. Among the latter are two of special importance. The first is obtained from (3.10) by replacing $k$ with $k+1$ and using (2.13):

$$
B g_{k} \equiv[(d / d x)+1][t(d / d x)+x][(d / d x)+1] g_{k}=(k+1) g_{k+1} .
$$

The other is obtained by differentiating (3.10) with respect to $x$, using (2.13) to express the right side in terms of $g_{k-1}$, and multiplying by $e^{x}$ before integrating:

$$
B^{\prime} g_{k} \equiv(d / d x)[t(d / d x)+x] d g_{k} / d x=k g_{k-1} \text {. }
$$

This last result depends on the appropriate choice of the constant of integration. We shall find a use for these relations in the group-theoretical considerations of the next section.

The diffusion polynomials $p_{k}(x)$ appearing as coefficients of $E(x)$ in (2.17) must satisfy not only the same recurrence relation as the $g_{k}(x)$ but also the differential recurrence relation (2.13); it follows that, like $g_{k}(x), p_{k}(x)$ satisfies the differential equation $A p_{k}(x)=k p_{k}(x)$, with $A$ given by (3.10). These remarks do not, however, extend to the polynomials $q_{k}(x)$.

Because of the close relation between the diffusion equation and the heat equation, it might be expected that there should be a relation between the diffusion polynomials and the heat polynomials which (see [25]) may be defined by

$$
v_{k}(x)=\exp \left[\frac{1}{2} t(d / d x)^{2}\right] x^{k} / k !
$$

where the exponential is to be replaced by its expansion in series. In fact the $p_{k}(x)$, like the $v_{k}(x)$, satisfy the diffusion equation without flow:

$$
C p_{k}=\partial p_{k} / \partial t-\frac{1}{2} \partial^{2} p_{k} / \partial x^{2}=0
$$

this is easily proved by noticing that $p_{0}$ and $p_{1}$, as shown by (3.8), satisfy the equation, and that the differential operator $C$ commutes with the operator $B$ in (3.11), which connects the contiguous polynomials $p_{k}$ and $p_{k+1}$. But, instead of (3.13), the $p_{k}(x)$ are given by

$$
p_{k}(x)=\exp \left[\frac{1}{2} t(d / d x)^{2}\right] L_{k}(-x) .
$$

This again is easily proved by noticing that it is satisfied for $k=0$ and $k=1$, and that the third order recurrence relation like (3.7) satisfied by the $p_{k}(x)$ follows from the second order recurrence relation (3.2) satisfied by the Laguerre polynomials. It follows from (3.15) that $p_{k}(x)$ is the solution of (3.14) for $t>0$, corresponding to an initial value $L_{k}(-x)$, in the interval $-\infty<x<\infty$. Finally, 
from (3.3), (3.13) and (3.15), we see that the relation between the diffusion polynomials and the heat polynomials can be expressed in the form

$$
p_{k}(x)=e^{-x}(d / d x)^{k}\left[e^{x} v_{k}(x)\right] .
$$

In spite of the presence of the exponential factors, both sets of polynomials satisfy the diffusion equation without flow $(v=0)$, because the differential operators $\exp \left[\frac{1}{2} t(d / d x)^{2}\right]$ and $e^{-x}(d / d x)^{k} e^{x}$ commute.

\section{Groups defined on images}

The differential operators $A, B$ and $B^{\prime}$ introduced in (3.10), (3.11) and (3.12) define a representation of the pseudo-orthogonal group $S O(2,1)$. That this is so follows from the commutation relations

$$
\begin{aligned}
{[A, B] } & =B, \\
{\left[A, B^{\prime}\right] } & =-B^{\prime}, \\
{\left[B^{\prime}, B\right] } & =2 A+1,
\end{aligned}
$$

which show that $A^{\prime}=A+\frac{1}{2}, B$ and $B^{\prime}$ constitute a basis in a representation of the Lie algebra of $S O(2,1)$. The Casimir invariant is $B^{\prime} B-\left(A^{\prime}\right)^{2}-A^{\prime}$, which reduces here to the multiple $\frac{1}{4}$ of the identity. Representations are carried by linear spaces with basis functions $\phi_{0}, \phi_{1}, \phi_{2}, \ldots$ satisfying

$$
\begin{gathered}
A \phi_{k}=k \phi_{k}, \quad B^{\prime} \phi_{k}=k \phi_{k-1}, \\
B \phi_{k}=(k+1) \phi_{k+1},
\end{gathered}
$$

and we have seen that the diffusion functions $g_{k}(x)$ and the diffusion polynomials $p_{k}(x)$ are examples of functions forming such sequences. From the spectrum of eigenvalues $k+\frac{1}{2}$ of $A^{\prime}$ and the value of the Casimir invariant, we deduce that it is the unitary representation $D^{+}(-1 / 2)$ of $S O(2,1)$ that is involved here, in the notation of Barut and Fronsdal [3]. There are many other examples of such sequences of functions satisfying differential equations of the second order, and defining representations of $S O(2,1)$ (see [15], [14], [11] and [2]). These include the Laguerre polynomials. But it is quite unusual to have two distinct sequences associated with the same differential equation, because, with analogous second order differential operators $A, B$ and $B^{\prime}$ satisfying (4.1), the equations $A \phi_{0}=0$ and $B^{\prime} \phi_{0}=0$ can have only one solution. Similarly, although there is a third sequence of functions $h_{k}$ satisfying both the recurrence relation (3.7) and the differential equation (3.10), it is not perfect in that (3.11) is not satisfied. However, that sequence may be of interest in providing solutions to the diffusion 
equation, and for comparison with (2.17) we give it:

$$
\begin{gathered}
h_{k}(x)=p_{k}(x) F(x)+t q_{k}(x) F^{\prime}(x) \\
F(x)=\int_{0}^{x} \int_{0}^{y} \exp \left[\frac{1}{2}\left(z^{2}-y^{2}\right) / t-z\right] d z d y .
\end{gathered}
$$

We now recall the relation between the sequence of diffusion functions $g_{k}$ and the sequence of images introduced in modelling the fluid reactor. There the functions $g_{0}$ represented the primary source, on the boundary of the reactor, and the sequence was constructed in such a way that $g_{k+1}$ represented the image of the source represented by $g_{k}$. From this standpoint, we see that the ladder operators $B$ and $B^{\prime}$ appearing in (4.1) have the effect of moving between any source and its image. In the enveloping algebra of the Lie algebra of $S O(2,1)$, there are linear operators of the type $B^{m}$ and $B^{\prime m}$ which effect transfers between arbitrary pairs of images. Furthermore, representatives of actual elements of the group $S O(2,1)$ can be constructed, as

$$
e\left(a, b, b^{\prime}\right)=\exp \left[i\left(a A+b B+b^{\prime} B^{\prime}\right)\right]
$$

(where $a$ is real, and $b$ and $b^{\prime}$ are complex conjugates). Thus we may regard the group as defined on the sequence of images of the fluid reactor. The importance of this group from the point of view of the theory of diffusion stems from the fact that it leaves invariant the partial differential operator $C$ of the diffusion equation as defined in (3.14):

$$
[C, A]=[C, B]=\left[C, B^{\prime}\right]=0 .
$$

As a result, from the fact that the first terms of the sequences $g_{k}(x), h_{k}(x)$ and $p_{k}(x)$, divided by $\sqrt{t}, t$ and 1 respectively, satisfy the diffusion equation, it follows that every term of those sequences will do the same.

In applications to specific problems like that considered in Section 2, the positions as well as the strengths of the images are important. Each image is related to its source by a reflection in the plane $x=0$ or $x=L$. If we denote these reflections by $R_{0}$ and $R_{L}$, then for any function $\phi(x)$

$$
\begin{gathered}
R_{0} \phi(x)=\phi(-x), \\
R_{L} \phi(x)=\phi(2 L-x) .
\end{gathered}
$$

We define

$$
g_{k}^{*}(x)=\exp \left(\frac{1}{2} x\right) g_{k}(x)
$$

and, with $B[=B(x)]$ as in (3.11),

$$
\begin{aligned}
B^{*}(x) & =\exp \left(\frac{1}{2} x\right) B \exp \left(-\frac{1}{2} x\right) \\
& =\left[(d / d x)+\frac{1}{2}\right]\left[t(d / d x)+x-\frac{1}{2} t\right]\left[(d / d x)+\frac{1}{2}\right],
\end{aligned}
$$

so that

$$
B^{*}(-x)=-\left[(d / d x)-\frac{1}{2}\right]\left[t(d / d x)+x+\frac{1}{2} t\right]\left[(d / d x)-\frac{1}{2}\right]
$$


Then we may write, using (3.11),

$$
\begin{array}{r}
g_{k+1}^{*}\left(x-y_{k+1}+t\right)=B^{*}\left(x-y_{k+1}+t\right) R_{0} g_{k}^{*}\left(y_{k}-x+t\right) /(k+1) \\
k \text { odd, } \\
g_{k+1}^{*}\left(y_{k+1}-x+t\right)=B^{*}\left(y_{k+1}-x+t\right) R_{L} g_{k}^{*}\left(x-y_{k}+t\right) /(k+1) \\
k \text { even, }
\end{array}
$$

with the $y_{k}$ defined as in (2.6). Defining the operator $T$ recursively by

$$
\begin{gathered}
T^{0} g_{0}^{*}(x+t)=g_{0}^{*}(x+t), \\
T^{k+1} g_{0}^{*}(x+t)= \begin{cases}B^{*}\left(x-y_{k+1}+t\right) R_{0} T^{k} g_{0}^{*}(x+t), & k \text { odd } \\
B^{*}\left(y_{k+1}-x+t\right) R_{L} T^{k} g_{0}^{*}(x+t), & k \text { even, }\end{cases}
\end{gathered}
$$

we can express the entire Green's function defined in (2.5), (2.7) and (2.8) in the compact form

$$
G(x, t)=-2 a(x, t)(\partial / \partial x)\left[\exp (-T) \exp \left(\frac{1}{2}(x+t)\right) g_{0}(x+t, t)\right],
$$

noting that (3.1) still holds here.

The group-theoretical significance of many properties of the special functions of the hypergeometric (including confluent hypergeometric) type is well documented (see Miller [15]; Green and Triffet [14]; Dieudonné [11]; Askey, Koornwinder and Schemp [2]). The considerations of this and the previous section point to the fact that many of these properties are shared by some functions satisfying differential equations of the third order, but that the latter have some interesting properties of their own.

So far in this paper we have chosen to consider the simplest type of linear reactor. However, our analysis is easily extended to a semi-permeable rectangular tank containing reacting material, placed obliquely to the direction of flow. Assuming that the velocity is uniform within the tank, it can be resolved into uniform components $v_{x}$ and $v_{y}$ parallel to the sides of the tank, and the diffusion pattern within the tank can be modelled by treating the fluxes in the two orthogonal directions independently. To take account of Danckwerts' boundary conditions, the primary sources on the boundary will be supplemented by a doubly infinite set of images extending over a plane containing the reactor. It is clear that the group defined on this set of images will be $S O(2,1) \times S O(2,1)$, locally isomorphic to $S O(2,2)$. If the tank is not rectangular but polygonal, the system of images and the group defined on those images will be even more complex. It is possible to extend these considerations also to the corresponding three-dimensional problems.

In the next section we shall present another type of generalisation of the diffusion functions and their associated polynomials. 


\section{A generalisation}

Because of the connection between the diffusion polynomials and the Laguerre polynomials, there is a further generalisation, involving a parameter $\alpha$, which could also be of interest in problems of diffusion, and which will allow us to define other representations of the groups defined on the system of images. As the relevant generalisation of the functions $g_{k}$, we introduce

$$
g_{k}^{\alpha}(x)=\int_{x}^{\infty} \exp \left(-\frac{1}{2} y^{2} / t\right) L_{k}^{\alpha}(y-x) d y,
$$

where the $L_{k}^{\alpha}(x)$ are associated Laguerre polynomials which for integral values of $\alpha$ are related to the Laguerre polynomials by

$$
L_{k}^{\alpha}(x)=(-d / d x)^{\alpha} L_{k+\alpha}(x) .
$$

They obviously satisfy the same differential recurrence relation (2.13) as the $L_{k}(x)$, and a similar recurrence relation

$$
(k+1) L_{k+1}^{\alpha}=(x+2 k+\alpha+1) L_{k}^{\alpha}-(k+\alpha) L_{k-1}^{\alpha} .
$$

In consequence, the $g_{k}^{\alpha}(x)$ satisfy the third-order recurrence relation

$$
\begin{aligned}
(k+1) g_{k+2}^{\alpha}= & (x+3 k+\alpha+4) g_{k+1}^{\alpha}-(x+3 k+2 \alpha+2-t) g_{k}^{\alpha} \\
& +(k+\alpha) g_{k-1}^{\alpha},
\end{aligned}
$$

and the third-order differential equation

$$
A_{\alpha} g_{k}^{\alpha} \equiv[[(d / d x)+1][t(d / d x)+x]+\alpha] d g_{k}^{\alpha} / d x=k g_{k}^{\alpha}
$$

By a generalisation of the methods adopted in Section 3, we can show also that if

$$
B_{\alpha}=[(d / d x)[t(d / d x)+x]+\alpha](d / d x),
$$

and

$$
B_{\alpha}^{\prime}=[[(d / d x)+1][t(d / d x)+x]+\alpha][(d / d x)+1]
$$

then

$$
\begin{aligned}
& B_{\alpha} g_{k}^{\alpha}=(k+\alpha) g_{k-1}^{\alpha}, \\
& B_{\alpha}^{\prime} g_{k}^{\alpha}=(k+1) g_{k+1}^{\alpha},
\end{aligned}
$$

so that $B_{\alpha}$ and $B_{\alpha}^{\prime}$ are the ladder operators in this extension. The operators $B_{\alpha}, B_{\alpha}^{\prime}$ and $A_{\alpha}^{\prime}=A_{\alpha}+\frac{1}{2} \alpha+\frac{1}{2}$ are $S O(2,1)$ generators, satisfying relations like (4.1), and the Casimir invariant is in this instance the multiple $\left(1-\alpha^{2}\right) / 4$ of the identity. The representation of $S O(2,1)$ involved depends on the value of $\alpha$.

There are also polynomials $p_{k}^{\alpha}(x)$ which satisfy the same differential equation and differential recurrence relations as $g_{k}^{\alpha}(x)$; these could be defined by an 
equation like (2.17), but at this point we shall clarify the relation between the diffusion polynomials and the associated Laguerre polynomials by writing

$$
p_{k}^{\alpha}(x)=\exp \left[\frac{1}{2} t(d / d x)^{2}\right] L_{k}^{\alpha}(-x) .
$$

It is obvious from this formula that the generalised diffusion polynomials also satisfy the diffusion equation.

We now notice the operator identity

$$
\exp \left[\frac{1}{2} t(d / d x)^{2}\right] x \exp \left[-\frac{1}{2} t(d / d x)^{2}\right]=t(d / d x)+x,
$$

which enables us to transform the recurrence relation (5.3) into a differential recurrence relation

$$
(k+2) p_{k+1}^{\alpha}=[t(d / d x)+x+2 k+\alpha+1] p_{k}^{\alpha}-(k+\alpha) p_{k-1}^{\alpha} .
$$

It is also clear from (5.9) that the $p_{k}^{\alpha}(x)$ satisfy the same relation

$$
(d / d x)\left(p_{k+1}^{\alpha}-p_{k}^{\alpha}\right)=p_{k}^{\alpha}
$$

as $L_{k}^{\alpha}(-x)$, so by elimination of the derivatives between (5.11) and (5.12) we have the third-order recurrence relation

$$
\begin{aligned}
(k+2) p_{k+2}^{\alpha}= & (x+3 k+\alpha+4) p_{k+1}^{\alpha}-(x+3 k+2 \alpha+2-t) p_{k}^{\alpha} \\
& +(k+\alpha) p_{k-1}^{\alpha} .
\end{aligned}
$$

By differentiating this recurrence relation and using (5.12), we verify that $p_{k}^{\alpha}(x)$ satisfies the same differential equation (5.5) as $g_{k}^{\alpha}(x)$, and that the third-order differential recurrence relations (5.8) are also satisfied when $g_{k}^{\alpha}$ is replaced with $p_{k}^{\alpha}$.

The relation (5.9) between the generalised diffusion polynomials and the associated Laguerre polynomials, which is in fact a similarity transformation, also gives insight into their connection with group theory. As remarked in the last section, the group theoretical significance of many properties of the special functions of hypergeometric and confluent hypergeometric type is well known, and the fact that the generalised diffusion polynomials are so related to polynomial solutions of the latter ensures that they inherit these properties. But their relation to the generalised diffusion functions $g_{k}^{\alpha}(x)$ is a special feature of the third-order equation which they satisfy, and may be an indication of the existence of still wider applications of group theory to the special functions of applied mathematics and mathematical physics.

\section{Appendix: An equivalent problem in heat conduction}

The problem considered in Section 2 is to find $c(x, t)$ for $0 \leq x \leq L$ and $t>0$, satisfying (1.3), the initial condition

$$
c(x, 0)=0 \text { for } 0<x<L,
$$


and the boundary conditions

$$
\begin{gathered}
c^{\prime}(L, t)=0, \quad \text { for } t>0, \\
v c(0, t)-D c^{\prime}(0, t)=j(0, t), \quad \text { for } t>0 .
\end{gathered}
$$

If we set

$$
u(x, t)=\exp \left[\left(\rho+v^{2} / 4 D\right) t-v x / 2 D\right] c(x, t),
$$

the problem becomes: find $u(x, t)$ for $0 \leq x \leq L$ and $t>0$ satisfying

$$
\begin{gathered}
\partial u(x, t) / \partial t=D u^{\prime \prime}(x, t) \quad \text { for } 0<x<L, t>0 \\
u(x, 0)=0 \text { for } 0<x<L \\
-D u^{\prime}(L, t)-\frac{1}{2} v u(L, t)=0 \text { for } t>0 \\
-D u^{\prime}(0, t)+\frac{1}{2} v u(0, t)=J(t) \text { for } t>0
\end{gathered}
$$

where

$$
J(t)=\exp \left[\left(\rho+v^{2} / 4 D\right) t\right] j(0, t) .
$$

Then $u$ can be interpreted as the temperature in a region with thermal diffusivity $D$, with a given influx of heat $J(t)$ at $x=0$, and "outer heat conduction" [19] at both ends, taking account of radiation losses etc.

It is a standard result [10] that the solution of this equivalent problem can be written in the general form

$$
u(x, t)=\int_{0}^{t} G^{*}\left(x, 0, t-t^{\prime}\right) J\left(t^{\prime}\right) d t^{\prime},
$$

where $G^{*}(x, y, t)\left[=G^{*}(y, x, t)\right]$ is, for $x, y \in[0, L]$ and $t>0$, a Green's function for the problem, satisfying (6.5) and (6.7) for each $y,(6.8)$ with $J(t)=0$ for each $y$, and

$$
\lim _{t \rightarrow 0+} G^{*}(x, y, t)=\delta(x-y) \quad \text { for } x, y \in(0, L) .
$$

We could not find the determination of this Green's function in the literature, but the corresponding problem for a semi-infinite region was solved a long time ago by Sommerfeld $\left[19\right.$, p. 67]. There one seeks a function $G_{0}^{*}(x, y, t)$ for $x, y \geq 0$, $t>0$, satisfying (6.5) and (6.11) for $x, y>0$, the boundary condition

$$
\frac{1}{2} v G_{0}^{*}(x, y, t)-D \partial G_{0}^{*}(x, y, t) / \partial x=0 \text { for } x=0 \text { and } y, t>0,
$$

and also $G_{0}^{*}(x, y, t) \rightarrow 0$ as $x \rightarrow \infty$ for $t>0$. Then defining $u(x, t)$ for $x \geq 0$, $t>0$ by (6.10) with $G_{0}^{*}$ replacing $G^{*}$, one obtains a solution of (6.5) and (6.6) on the semi-infinite region, and of (6.8), satisfying $u(x, t) \rightarrow 0$ as $x \rightarrow \infty$. Inverting (6.4), one then has a solution $c(x, t)$ of (1.3) and (6.1) for $x, t \geq 0$, satisfying (6.3), in the form

$$
c(x, t)=\int_{0}^{t} G_{0}\left(x, t-t^{\prime}\right) j\left(0, t^{\prime}\right) d t^{\prime}
$$


where

$$
G_{0}(x, t)=\exp \left[v x / D-\left(\rho+v^{2} / 4 D\right) t\right] G_{0}^{*}(x, 0, t) .
$$

But this function $G_{0}$ is precisely the first term in the series (2.5), since it evidently corresponds to the concentration arising from an influx $j(0, t)=\delta(t)$ when the length $L$ of the region goes to infinity; and at any finite positive value of $x$, the contributions of all images $G_{1}, G_{2}, \ldots$ must go to zero in that limit.

According to Sommerfeld

$$
\begin{aligned}
G_{0}^{*}(x, y, t)= & (4 \pi D t)^{-1 / 2}\left[\exp \left[-(x-y)^{2} / 4 D t\right]+\exp \left[-(x+y)^{2} / 4 D t\right]\right. \\
& \left.-(v / D) \exp (v y / 2 D) \int_{-\infty}^{-y} \exp \left[v \eta / 2 D-(x-\eta)^{2} / 4 D t\right] d \eta\right]
\end{aligned}
$$

so that

$$
\begin{aligned}
G_{0}^{*}(x, 0, t)= & (\pi D t)^{-1 / 2}\left[\exp \left(-x^{2} / 4 D t\right)\right. \\
& \left.-(v / 2 D) \int_{x+v t}^{\infty} \exp \left[(v / 2 D)(x+v t-y)-(y-v t)^{2} / 4 D t\right] d y\right],
\end{aligned}
$$

and hence

$$
\begin{aligned}
G_{0}(x, t)= & (\pi D t)^{-1 / 2} \exp (-\rho t)\left[\exp \left[-(x-v t)^{2} / 4 D t\right]\right. \\
& \left.-(v / 2 D) \exp (v x / D) \int_{x+v t}^{\infty} \exp \left(-y^{2} / 4 D t\right) d y\right]
\end{aligned}
$$

Our construction of $G$ from $G_{0}$ using images, as in (2.5), (2.7) and (2.8), can be seen to provide also a solution of the problem of heat conduction posed by (6.5)-(6.8): the function $G^{*}(x, 0, t)$ is evidently given in terms of images by

$$
\begin{aligned}
G^{*}(x, 0, t) & =\exp \left[\left(v^{2} / 4 D+\rho\right) t-v x / 2 D\right] G(x, t) \\
& =\exp \left[\left(v^{2} / 4 D+\rho\right) t-v x / 2 D\right] \sum_{k=0}^{\infty} G_{k}(x, t),
\end{aligned}
$$

and the required solution is then given by $(6.10)$.

\section{Acknowledgement}

We are grateful to the Australian Research Grants Committee for the award of a Research Fellowship to one of us (H.S.G.). 


\section{References}

[1] M. Abramowitz and I. A. Stegun, Handbook of Mathematical Functions (U.S. Nat. Bur. of Standards, Washington, 1964).

[2] R. A. Askey, T. H. Koornwinder and W. Schemp (eds.), Special Functions: Group Theoretical Aspects (D. Reidel, Dordrecht, 1984).

[3] A. O. Barut and C. Fronsdal, Proc. Roy. Soc. London Ser. A 287 (1965) 532-548.

[4] L. Bass, A. J. Bracken and H. S. Green, sub. Chem. Engng. Sci. (1987).

[5] G. W. Bluman and J. D. Cole, J. of Math. and Mech., 18 (1969) 1025-1042; see also G. W. Bluman and J. D. Cole, Similarity Methods for Differential Equations (Springer, Berlin: Applied Mathematical Sciences Series 13, 1974).

[6] A. R. van Cauwenberghe, Chem. Engng. Sci., 21 (1966) 203-205.

[7] P. V. Danckwerts, Chem. Engng. Sci., 2 (1953) 1-13.

[8] W. Deckwer and E. A. Mählmann, Adv. Chem., 133 (1974) 334-347.

[9] W. Deckwer and E. A. Mählmann, Chem. Engng. J., 11 (1976) 19-25.

[10] R. Dennemeyer, Introduction to Partial Differential Equations and Boundary Value Problems (McGraw-Hill, New York, 1968).

[11] J. A. Dieudonné, Special Functions and Linear Representations of Lie Groups (Amer. Math. Soc., Providence, R.I., 1980).

[12] A. Erdelyi (ed.), Bateman Manuscript Project Vol. 1 (1953) (McGraw-Hill, New York, 1953).

[13] H. S. Green, "Pollution by Diffusive Processes". Pollution: Engineering and Scientific Solutions (ed. E. S. Barrekette) (Plenum, New York, 1973).

[14] H. S. Green and T. Triffet, J. Math. Phys. 10 (1969) 1069-1089.

[15] W. Miller, Lie Theory and Special Functions (Academic Press, New York, 1968).

[16] S. J. Parulekar and D. Ramakrishna, Chem. Engng. Sci., 39 (1980) 1571-1579, 1581-1597, 15991611.

[17] W. Perl and F. P. Chinard, Circulation Research 22 (1968) 273-298.

[18] M. S. Roberts and M. Rowland, J. Pharmacokinet. Biopharm. 14 (1986) 227-308.

[19] A. Sommerfeld, Partial Differential Equations in Physics (Academic Press, New York, 1949).

[20] S. Sundaresan, N. R. Amundson and R. Aris, AIChE J., 26 (1980) 529-536.

[21] A. M. Vandenberg and M. A. B. Deakin, History of Mathematics Papers 36 (Maths. Dept., Monash Univ., 1986) 1-96.

[22] J. F. Wehner and R. H. Wilhelm, Chem. Engng. Sci., 6 (1956) 89-93.

[23] C. Y. Wen and L. T. Fan, Models for Flow Systems in Chemical Reactors (M. Dekker, New York, 1975).

[24] E. Wicke, Chemie-ing.-Techn. 47 (1975) 547-551.

[25] D. V. Widder, The Heat Equation (Academic Press, New York, 1975). 